\title{
Poétique et imaginaires de Montréal dans la littérature québécoise contemporaine
}

\author{
Poetics and Imaginaries of Montreal in Contemporary Quebec \\ Literature
}

Adina Balint ${ }^{1}$

Submetido em 5 e aprovado em 11 de novembro de 2017.

Résumé: Le récit littéraire d'aujourd'hui ne semble plus habiter une ville, mais un palimpseste de villes. «L'esprit migrateur» (Pierre Ouellet) et la rencontre transculturelle» (Patrick Imbert) s'avèrent être les nouvelles conditions de l'imaginaire contemporain. Quels visages de Montréal se déplient dans La Québécoite de Régine Robin et dans La femme qui fuit d'Anaïs Barbeau-Lavalette? Comment peut-on arpenter Montréal, se l'approprier, en saisir les secrets? Et quels rapports à la transculture? Si le thème du déplacement s'est largement répandu dans la littérature québécoise depuis les années 1980, sous l'impulsion des écrivains issus de la migration, aujourd'hui, le déplacement n'est plus d'ordre géoculturel, mais de nature symbolique et ontologique.

Mots-clés: Montréal. Littérature québécoise contemporaine. Imaginaire. Régine Robin. Anaïs Barbeau-Lavalette.

\begin{abstract}
Contemporary narratives no longer display a singular city but a palimpsest of cities. "L'esprit migrateur" (Pierre Ouellet) and "la rencontre transculturelle" (Patrick Imbert) inhabit the new literary imaginary. What representations of Montreal do texts, such as La Québécoite by Régine Robin and La femme qui fuit by Anaïs Barbeau-Lavalette present? How can we describe the experience of wandering through the city of Montreal? And what relationships with transculturalism? If the topic of mobility has been common in Quebecois literature since the 1980s, mainly in the works of migrant writers, mobility is not geocultural any more today: it is rather symbolic and ontological.
\end{abstract}

Keywords: Montreal. Contemporary Quebecois literature. Imaginary. Régine Robin. Anaïs Barbeau-Lavalette.

\section{Introduction}

La ville anime l'imaginaire littéraire québécois des années 1950 jusqu'à aujourd'hui, tous genres confondus. Au début des années 1950, l'espace urbain apparaît souvent dans le roman comme toile de fond pour évoquer la détresse d'un monde où 
les modèles anciens deviennent graduellement caducs ou bien pour exprimer des désirs de renouvellement idéologique et politique (Gabrielle Roy, Bonheur d'occasion, Les Automatistes, Refus global). Dans les années 1960-1970, l'imaginaire de la ville accueille l'idée nationale, l'espérance communautaire et l'élan féministe, se proposant comme espace-support d'une réflexion politique, sociale et culturelle militante, sur arrière-plan d'humiliation des francophones (Antonine Maillet, Pélagie-la-Charrette, Gabrielle Roy, La Route d'Altamont). Mais la ville peut aussi représenter la scène subversive d'une parole ordinaire, voire populaire (joual ou chiac), puis, dans les années 1980-1990, le signe d'une ouverture à des identités plurielles (France Daigle, Michel Tremblay, Régine Robin). Enfin, avec le nouveau siècle, la représentation de la ville ouvre à une autoréflexivité du sujet, d'un sujet saisi dans ses multiples dimensions, psychique, sociale, éthique (Dany Laferrière, L'énigme du retour, Ying Chen, Quatre mille marches, Anaïs Barbeau-Lavalette, La femme qui fuit $)^{2}$.

La ville de Montréal, en particulier, hante depuis toujours l'imaginaire québécois dès les récits de fondation jusqu'aux récits contemporains. Prenons un exemple de la littérature dite «migrante»: en 1983, Régine Robin publie chez Québec Amérique son roman La Québécoite, que la critique reconnaît comme un roman emblématique de l'écriture postmoderne, qui travaille le décentrement, les questions d'adaptation et la confrontation entre les cultures. À l'époque, quand Robin faisait ses allers et retours entre Paris et Montréal, par cette fiction, elle cherchait à inscrire la grande ville dans l'écriture, une grande ville qu'elle découvrait en tant que nouvelle arrivée au Québec: elle n'est pas encore Canadienne, n'a pas le droit de vote, mais elle s'intéresse au discours social québécois, à la littérature du Québec (elle émigre à Montréal en 1977; en 1979, elle publie Le Cheval blanc de Lénine chez XYZ éditeur). Ce qui la retient avant tout, c'est Montréal, la grande ville, la ville cosmopolite où tout lui paraît étrange, curieux, nouveau et possible. Une ville des recommencements. Il existe déjà de nombreux livres qui prennent Montréal comme objet, comme thématique, en se penchant sur un quartier ou sur une rue (Robert de Roquebrune, Quartier Saint-Louis, 1966; André Carpentier, Rue SaintDenis, contes fantastiques, 1978), mais Robin n'y trouve pas l'écho de la fragmentation et de l'hétérogénéité qui la frappent. C'est la Montréal postmoderne qu'elle tente de nous 
livrer dans La Québécoite: une ville et une écriture de la ville qui valorisent la pluralité, l'hybridité, le fragment, le métissage, voire l'éclatement - des visées qui sont aussi celles du groupe d'intellectuels, d'écrivains et de penseurs rassemblés autour de la revue Vice Versa (1980-1996).

La ville de Montréal fascine aussi les chercheurs en sciences humaines qui entament des projets à ce sujet, écrivent des livres et des articles, organisent des colloques. Le site de la Bibliothèque et Archives nationales du Québec, à la section Culture, contient une liste de références bibliographies au sujet de: «Écrire Montréal, la ville dans la littérature». ${ }^{3}$ Retenons également le Laboratoire sur les Récits du soi mobile (fondé en 2012; http://www.lrsm.ca), dirigé par Simon Harel, avec un réseau de chercheurs et partenaires culturels et médiatiques, qui se penchent sur des questions d'espace, urbanité, mobilité, récit, sujet...

En 1992, Pierre Nepveu et Gilles Marcotte ont co-dirigé un ouvrage collectif intitulé Montréal imaginaire. Ville et littérature, paru aux éditions Fides. L'ouvrage regroupe sept études et adopte une perspective d'ensemble historique pour mettre en lumière l'ambivalence des auteurs québécois face à la ville. Le livre est structuré en plusieurs parties: Quelques récits de fondation de Montréal - La ville dans le roman populaire du 19e siècle - Poésie et roman au tournant du siècle - Montréal dans la prose narrative des années 1934-1936 - La présence américaine dans le roman montréalais (1945-1970) - Montréal dans la poésie québécoise contemporaine - La parole orpheline de l'écrivain migrant. Dans le texte de l'introduction «Montréal, sa littérature», Nepveu et Marcotte écrivent:

Il est évident que, sans Montréal, la littérature québécoise n'existe pas. Montréal, c'est l'institution littéraire elle-même: ses instances, l'édition, la critique, les académies, la plupart des écrivains, mais aussi la source même d'une certaine idée de la littérature. Québec, d'autres villes aussi jouent des rôles importants; que serait l'œuvre d'un Jacques Poulin sans la mythologie fascinante du Vieux Québec? Mais Montréal ne s'appelle pas indûment la métropole, la ville-mère, la ville-marie. Montréal n'est pas tout; elle est peut-être l'image du tout.

La littérature québécoise n'existe pas sans Montréal; peut-on dire que Montréal n'existe pas sans la littérature? Pour les autres grandes villes du monde, il semble que la représentation littéraire 
soit indispensable au constat d'existence. Paris c'est Balzac (et quelques autres); Londres, Dickens ; Berlin, Döblin; New York, Dos Passos; Dublin, Joyce, et ainsi de suite. Aucun nom d'écrivain n'est associé de façon aussi nécessaire, aussi immédiate, à Montréal. $\mathrm{Ni}$ anglais, ni français. Car cela ne simplifie pas les choses, il existe bien deux littératures à Montréal, et si la française est la plus nombreuse, il n'est pas sûr que plusieurs des images les plus fortes de la métropole ne soient pas venues de l'autre côté de la barrière linguistique. Mais ni Gabrielle Roy, ni Hugh McLennan, ni Mordecai Richler, ni Réjean Ducharme, pour rester chez les romanciers, ne se sont approprié le label montréalais. Ils sont propriétaires d'une rue, de quelques rues, d'un quartier, plutôt que de la ville même. (...)

Une ville existe, littérairement, lorsqu'elle devient une question, lorsqu'elle fait question ( $p .7-8$ ).

Nepveu et Marcotte soulignent que Montréal n'est devenue une question, pour la littérature, qu'aux environ des années soixante, c'est-à-dire à partir de ce qu'on a appelé la Révolution tranquille. Autrefois, elle existait, mais plutôt comme symbole d'un progrès spirituel ou matériel.

À partir des années 1980, cette réflexion sur la ville est enrichie par les écritures dites migrantes ou transmigrantes que Régine Robin définit dans ces termes: «écriture transmigrante: quand les intertextes et les références culturelles s’échangent véritablement ou se rencontrent, ne serait-ce que dans le malentendu ou le dialogisme». ${ }^{4}$ Les dynamiques urbaines hétéroclites de Montréal sont ainsi saisies à travers l'exploration des identités et des langues, du devenir minoritaire, de la rencontre et de l'altérité chez des écrivains comme Dany Laferrière dans Comment faire l'amour avec un nègre sans se fatiguer (1985) et plus récemment, dans Tout ce qu'on ne te dira pas, Mongo (2015), par Sergio Kokis, dans Le pavillon des miroirs (1994), par Ying Chen, dans Les lettres chinoises (1993), Kim Thuy, dans Ru (2009) ou Mauricio Segura, Côte-des-Nègres (1998), par exemple.

\section{Dynamiques urbaines hétéroclites dans La Québécoite}

Revenons à La Québécoite de Régine Robin. Dans un premier temps, la narratrice met en scène un personnage féminin, une immigrante arrivée de Paris, le double de l'écrivaine, mais pas tout à fait, parce que le texte demeure fictionnel. Ce personnage habite successivement trois quartiers de Montréal, où elle s'établit avec un compagnon. 
Chaque quartier symbolise un devenir possible de sa vie montréalaise: Snowdon, Outremont et Jean-Talon. Paris et Montréal se font écho. Robin utilise délibérément la structure du collage, l'effet de liste, l'inventaire, la description, la déambulation, formes les plus adéquates pour montrer l'étrangeté de Montréal, son caractère hétéroclite, sa fragmentation, son clivage. La ville est ici cet espace de repérage, de reconnaissance, de déchiffrement qu'elle a souvent offert, comme un livre ouvert, aux écrivains juifs Régine Robin, en est un exemple, malgré la terrible mémoire des rafles et des chambres à gaz, obstacle perpétuel à l'habitation paisible de tout lieu.

Le personnage, arrivé de Paris, part à la découverte de Montréal en se perdant constamment. Ses pérégrinations sont portées par un désir fort, celui d'appartenir à la ville, d'appartenir ici, ce qu'elle appelle dans son essai Nous autres, les autres (2011): «Je suis devenue d'ici» (p. 270):

La rencontre avec une ville. Tu te perdais souvent, revenant mille fois au même endroit, reconnaissant les enseignes, les boutiques, la qualité de l'air flottant autour des carrefours. Au bout d'un mois les itinéraires familiers avaient remplacé les déambulations hésitantes, maladroites des premiers temps. C'est au petit matin que les villes prennent couleur. Il y a aussi les villes sans couleur, teintées d'eau, de neige. Les villes brumes et sirènes, les villes cheminées d'usine, les villes parcs, les villes fleurs. Tu avais aimé toutes les villes, la respiration hallucinée des villes américaines vues d'avion, le soir comme un tableau de bord, un écrivain électronique de lignes croisées, des réseaux lumineux comme dans la nuit (p. 17).

Cet espace foisonnant pourrait évoquer la créolisation, conception revendiquée par des penseurs antillais tel Édouard Glissant, qui s'oppose dans une certaine mesure aux valeurs états-uniennes classiques, et, en tout cas, à ce qu'on appelle l'américanisation, touchant surtout la culture des médias et les modes de vie. La précipitation, sur une plage temporelle très courte, des contacts et des échanges entre cultures ferait en sorte, selon Glissant, que nous habitons désormais le «chaos-monde», ou le «tout-monde» ${ }^{5}$, faisant face perpétuellement à une nouveauté imprévisible qu'échoue à cerner et à comprendre la pensée de l'unité.

On note que le personnage féminin de Robin cherche un lieu où se sentir chez soi sans être enfermée dans un carcan territorial ou identitaire. Cette femme incarne 
les idées de la postmodernité et de la transculture: elle veut circuler, flotter librement, elle ne veut pas jouer de la posture de chacun dans son ghetto et sa spécificité - elle rejette la simple cohabitation tolérante dans le cadre du multiculturalisme que Robin et les intellectuels du Vice Versa pensaient à l'époque, «comme la juxtaposition mosaïque de ghettos sans liens les uns avec les autres» ${ }^{6}$. Le personnage de La Québécoite aspire aux «lignes croisées», à un vrai métissage culturel, une imprégnation des cultures, un processus de passage, de mouvement, de métamorphose. Elle est ouverte aux identités plurielles et à la transculture, telle que la définit Lamberto Tassinari: «Le trans [...] signifiait traversée, passage, métamorphose continue de l'identité, perte et gain sans arrêt, osmose». ${ }^{7}$ Cette prédilection au déplacement et à la recontextualisation rejoint la pensée sur le transculturel de Patrick Imbert, qui souligne la plus-value d' une mise en réseau des langues et des discours », « un métissage des formes et des genres». ${ }^{8}$ En effet, dans le récit de La Québécoite, la dynamique du déplacement autant géographique et symbolique du personnage croise les thèmes de l'altérité, de l'identité, de la transculture.

\section{Représentations contemporaines de Montréal: de La Québécoite à La femme qui fuit}

Que se passe-t-il en 2015, dans le récit d'Anaïs Barbeau-Lavalette, jeune auteure et cinéaste québécoise, qui publie une biofiction, La femme qui fuit, chez Marchand de feuilles? Le livre est très bien reçu par la critique, tiré à 75.000 exemplaires, couronné de plusieurs prix: le Prix littéraire France-Québec et le Prix des libraires du Québec 2016 et le Grand Prix du livre de Montréal. Il est aussi publié aux éditions Le Livre de Proche en France.

De quoi parle ce livre? Il restitue l'histoire d'une femme - la grand-mère de l'écrivaine, qu'elle n'a pas connue - en rapport avec la ville de Montréal. Cette femme, la grand-mère, s'appelle Suzanne Meloche. En 1948, elle est aux côtés de Borduas, Gauvreau et Riopelle quand ils signent Refus Global. Mais elle ne le signe pas. Avec Barbeau, elle fonde une famille. Mais très tôt, elle abandonne ses deux enfants. Pour toujours.

L'écrivaine Barbeau-Lavalette va à la rencontre de Suzanne Meloche-Barbeau, sa grand-mère. Ce faisant, elle tente de combler le vide laissé par celle-ci, lorsqu'elle a quitté mari et enfants. Ce retour au passé, de 1926 à 2009, nous fait voyager au Québec, en France, en Angleterre et aux États-Unis. Suzanne enfant est de celles qui a connu la 
crise économique et les contraintes de l'Église catholique au Québec. Adolescente, elle cherche à s'échapper de la morosité ambiante. C'est ainsi que son talent oratoire l'amène dans un concours auquel participe Claude Gauvreau. Plus tard, elle retrouve ce dernier, en compagnie de ses amis automatistes, lorsqu'elle vient terminer ses études à Montréal.

Voici comment la narratrice omnisciente de La femme qui fuit exprime quelque chose qui a changé dans son personnage, à la suite du retour de la métropole:

Tu reviens [de Montréal] donc chez toi en ébullition. Les jours reprennent leurs cours, mais tu les traverses autrement. Portée par le courant. Tu sais maintenant que tu as un ailleurs.

Ce que tu ne sais pas, c'est que tu en auras toujours un, et jamais le même. Ce sera ta tragédie (p. 87).

Cette expérience sensible du changement, de la différence, a quelque chose de mystérieux, et il faudra de la créativité ${ }^{9}$ au personnage pour décrypter son sens. Dans ce roman en particulier, la créativité est synonyme de fuite comme libération de toutes les contraintes: fuite d'Ottawa vers Montréal, fuite de Montréal en Gaspésie et puis, en Europe et à New York... départs et retours à travers lesquels le personnage se transforme, se métamorphose, grandit. Suzanne épouse Marcel Barbeau. Ils ont deux enfants, Manon et François. Les Barbeau et leurs amis s'installent à Saint-Hilaire, mais Suzanne se sent de plus en plus exclue du groupe. Déçue par l'échec du message libertaire du manifeste Refus Global (1948), elle quitte le foyer conjugal, en laissant la charge de ses enfants à son mari.

Il y a quelque chose de brisé dans l'âme de cette femme sa fragilité émotive et sentimentale la poursuivra d'ailleurs jusqu'à la fin de ses jours. Sa non-reconnaissance engendre son non-engagement, malgré son adhésion à diverses causes identitaires, dont celle défendue par un groupe antiségrégationniste au Sud des États-Unis. À l'instar de Deleuze, le changement est ressenti comme quelque chose de libérateur mais de douloureux à la fois: on croyait que tout resterait identique mais tout continue de changer. Le récit confirme de la sorte l'intuition deleuzienne que chaque moment de notre vie est entièrement différent des moments qui précèdent. Chaque moment est un nouveau commencement.

À la page 263 de La femme qui fuit, on lit ce qui suit à l'occasion d'un nouveau retour à Montréal: 
Un an seulement s'est écoulé, tu as l'impression d'années. Mais de retrouver ta ville te bouleverse. Tu n'aimes pas ce qui est fixe, ça te donne le vertige. Tu as si peur de reprendre racine. Tu dis à Peter que tu pars à nouveau. Il te répond qu'il te suit. (p. 248)

[...]

Montréal n'a pas changé. Mais après Londres, la ville te semble rajeunie. Puérile, inachevée. Cette candeur-là te fait du bien. Tu te sens débutante, toi aussi (p. 263).

En quelque sorte, ces paroles mettent en lumière que faire l'expérience du nouveau dans le sensible signifie voir que la vie est toujours ouverte, jamais close et déterminée entièrement. Rien n'est définitif et ne le sera jamais.

Dans un chapitre sur les années 1946-52, on lit:

Montréal a quelque chose de toi. (...). (p. 97)

Tu cherches à dire, tu cherches à faire. Tu te sens enfant dans un projet trop grand. Qui t'excite et t'effraie.

Vous [Claude et toi] arpentez la ville en tramway, sans but précis. Claude joue pour toi au guide touristique, te déballe sa ville à voix haute, comme un cadeau infini.

Vous sautez d'un tramway à l'autre, tu te glisses parmi les corps inconnus, te laisses envelopper de leur présence anonyme. [...] C'est ta façon de t'inscrire dans un nouveau paysage (p. 98-99).

On note ici la déambulation, la flânerie et le même désir de découvrir la ville comme un «nouveau paysage» que chez Régine Robin: la liberté et la renaissance intérieure et un éveil au monde extérieur. En même temps, lors de ce déplacement dans Montréal, il en émerge une réalité nouvelle. Dans la marche, il y a symboliquement le surgissement de l'inouï, de l'imprévu, de l'inattendu, du non perçu, d'un phénomène nouveau et indépendant.

Cela nous permet d'évoquer le concept de transculturation formulé par Fernando Ortiz, un Cubain qui cherchait à décrire le métissage complexe propre à la culture de Cuba. À partir d'Ortiz, Jean Larose ${ }^{10}$ écrit dans Vice Versa en 1987:

La transculturation est un ensemble de transmutations constantes: elle est créatrice et jamais achevée. Elle est toujours un processus dans lequel on donne quelque chose en même temps qu'on reçoit: les deux parties s'en trouvent modifiées. Il en émerge une réalité nouvelle qui n'est pas une mosaïque de caractères, mais un phénomène nouveau, original et indépendant (p. 19). 
L'écriture de la métamorphose de Suzanne Meloche et la créativité qu'elle a mise en acte au fil des différentes périodes de sa vie - du premier chapitre intitulé «1930-1946», jusqu'au dernier qui s'appelle «Aujourd'hui» - vient confirmer qu'il est possible de créer une «réalité nouvelle» à l'instar des dynamiques de la transculturation, tout en se créant soi-même: faire de sa vie une œuvre d'art, c'est-à-dire demeurer ouvert au fait que le monde est toujours nouveau, apprécier l'intensité des moments qui le traversent comme si c'était une «première fois». Cette première fois, on la ressent dans la voix narrative de La femme qui fuit, pour qui les transformations de l'intériorité résonnent avec l'espace urbain: «Montréal n’a pas changé. Mais après Londres, la ville te semble rajeunie. Puérile, inachevée. Cette candeur-là te fait du bien. Tu te sens débutante, toi aussi» (p. 263). Ni cauchemar, ni paradis, Montréal est ici la ville du temps perdu et retrouvé, la pensée s’y perd dans un travail de réminiscences, lorsque la femme-personnage se retrouve prise entre deux impossibilités: la chimérique résurgence du passé et la peur de se retrouver «fixée» par ce passé et les racines, d'où la fuite comme une issue salvatrice. Inconfortable chaos-monde.

Dans une démarche comparatiste, le récit de Robin et celui de Barbeau-

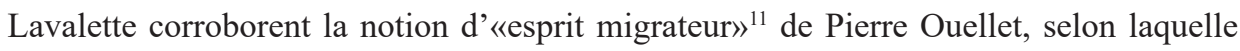
les thématiques de la migration et les déplacements géo-culturels et subjectifs ne sont plus explorés uniquement par des écrivains dits migrants, car «la migrance n’est pas seulement de nature géoculturelle, liée du déplacement d'un territoire à un autre ; elle est aussi et peut-être surtout de nature ontologique et symbolique, puisqu'elle caractérise le déplacement même du Sens et de l'Etre dans l'expérience intime de l'altérité». ${ }^{12}$ Cette même idée de la non-distinction entre les thématiques explorées par les écrivains de la migration et les écrivains issus du Québec apparaît également chez Maude Labelle dans un article sur Les lettres chinoises de Ying Chen:

Le récit ne serait pas migrant à cause de l'origine de son auteur, mais bien par le fonctionnement discursif qui rendrait compte du déplacement et de la différence, qui insisterait sur le jeu entre étrangéité et familiarité plutôt que sur l'un ou l'autre. Le brouillage des frontières, plutôt que les frontières elles-mêmes, deviendrait le point de rencontre des diverses pratiques des écrivains migrants, qu'ils soient d'ici ou d'ailleurs. ${ }^{13}$ 
C'est cette expérience intime de l'altérité et la mise en discours de la différence qu'explorent les personnages-narratrices de Robin et de Barbeau-Lavalette: autant par rapport à la ville de Montréal, qu'à travers l'acceptation du devenir-autre de soi. Ainsi, l'altérité apparaît dans ces récits comme une forme de vie, un ethos, une façon d'être au monde, dans un monde qui exige d'emblée une prise en compte de son altérité constitutive.

Revenons encore un instant à La Québécoite. Dans un deuxième temps, le même personnage rencontre à New York celui qui va devenir son mari, un intellectuel montréalais qui a fait son doctorat à New York et que son père, immigrant français, a envoyé à l'école anglaise pour «éviter les curé» (p. 46), selon sa propre expression. Ils font connaissance. Elle lui demande:

Vous vous sentez quoi au juste, Américain, Canadien, Québécois, Juif, Français? Ça a l'air compliqué cette histoire?

- En effet. Il aurait répondu pince-sans-rire, à la manière de Woody Allen - non ce n'est pas compliqué.

Je me sens

New-yorkais de Paris ou

Montréalais du Shtetl si vous voulez (p. 35).

Quand il s'agit d'identité, il est évident que les personnages, ainsi que les écrivains de la migration, jouent avec la pluralité et brouillent les frontières susceptibles de superposer langue, culture et identité. Montréal devient alors l'espace d'accueil de ces identités. Montréal est multiple elle-même.

Dans la postface de La Québécoite, lors de sa sortie en collection de poche en 1993, Robin s'est qualifiée «d'allophone d'origine française». Dany Laferrière se définissait plaisamment comme «un écrivain japonais»:

Dans quelle langue allez-vous écrire vos livres? Pas en anglais me dit le francophone. Pas en français me reproche à l'avance le créolophone. Tu ne trouveras pas de lecteur en créole, me lance le colonisé qui se croit réaliste... Personne ne me conseille simplement de donner le meilleur de moi-même, comme on le fait pour les sportifs. Moi qui croyais qu'on écrivait précisément pour effacer les frontières.... ${ }^{14}$

Ce qu'ils revendiquent, ces écrivains, c'est le droit de ne pas choisir! De vivre dans l'écriture leurs diverses identités imaginaires et réelles sans hiérarchie, sans ordre normatif. 
Cette représentation de l'identité plurielle de l'écrivain et de son double narrateur résonne avec la mise en discours de la ville comme «espace potentiel», pour reprendre une notion de Simon Harel, dans Les Passages obligés de l'écriture migrante. Cette ville composite fait figure de:

[...] sculpture en mouvement, une installation, un mobile qui donne la possibilité d'imaginer un « espace potentiel » : l'espace de négociation entre l'affirmation de l'individuel et la loi de la collectivité. En fait, la situation ne sait pas trop départager l'envers et l'endroit, le dedans et le dehors. Elle ne forme pas un habitat réel mais correspond plutôt à la demeure imaginaire du sens. ${ }^{15}$

La ville de Montréal donc: ouverte à la vraie diversité - celle qu'a pensée aussi Glissant, pas la diversité au sens péjoratif ${ }^{16}$ - que les gens de différentes cultures accueillent, et à laquelle ils sont sensibles, comme le montre un article de Pierre André Normandin, du 29 avril 2017 dans La Presse, intitulé «Une nouvelle publicité de Montréal retirée pour son manque de diversit» ${ }^{17}$.

\section{Conclusion}

Quelles sont les dynamiques des représentations littéraires de Montréal au vingtet-unième siècle dans les textes québécois? Du point de vue thématique: celles qui sont ouvertes au déplacement, à la différence, à la transculture comme échanges mutuels d'où surgit le nouveau, l'inédit - des sujets qui sont explorées par des écrivains issus du Québec et d'ailleurs. Du point de vue du genre, on assiste au vingt-et-unième siècle au triomphe du document: écriture d'enquête (la biofiction de Barbeau-Lavalette), biographie et autobiographie, écriture de voyage et autres formes de récits fragmentaires (Monique Proulx dans Ce qu'il reste de moi) refusant de se dire romans simplement. Or, ces écritures ne se contentent pas de déjouer les classements des bibliothèques, ils modifient profondément les catégories du littéraire et imposent une poétique et des imaginaires propres: exhibant l'auteur dans le récit, multipliant les narrateurs, créant des chapitres courts qui se mélangent avec des morceaux de lettres, de citations dans des compositions hybrides, par exemple, ces écritures contemporaines qui engagent les rapports entre la fiction et la réalité historique brouillent les genres du discours et nous 
conduisent à réfléchir aux distinctions disciplinaires opposant l'écrivain à l'historien, au journaliste et au sociologue.

Finalement, à travers les représentations de Montréal dans La Québécoite et dans La femme qui fuit, sommes-nous en train de saisir un nouveau désir de la littérature de témoignage, d'inventaire, d'information ou de documentation, visant à réinventer ses formes tout en cherchant à dire les multiples rapports - fragiles ou naturels, banals - que nous avons avec le monde?

\section{Référence}

. «Écrire Montréal, la ville dans la littérature». URL:http://www.banq.qc.ca/ collections/collections_patrimoniales/bibliographies/lire_montreal/culture1.html (page consultée le 31 octobre 2017).

BARBEAU-LAVALETTE, Anaïs. La femme qui fuit. Montréal: Marchand de feuilles, 2015. [Paris : Le Livre de Poche, 2015].

BORDUAS, Paul-Émile. Refus global et autres écrits. Montréal: Typo, 2010.

CACCIA, Fulvio Dir. La Transculture et Vice Versa. Montréal: Triptyque, 2010.

CARPENTIER, André. Rue Saint-Denis, contes fantastiques. Montréal: HMH, 1978.

CHEN, YING. Les lettres chinoises. Montréal: Boréal, 1993.

CHEN, Ying. Quatre mille marches. Montréal: Boréal, 2004.

DELEUZE, Gilles. Différence et répétition. Paris: Presses universitaires de France, 1968.

GLISSANT, Édouard. Poétique de la relation. Paris: Gallimard, 1990.

HAREL, Simon. Les Passages obligés de l'écriture migrante. Montréal: XYZ éditeur, 2005.

IMBERT, Patrick (dir.). Le transculturel et les littératures des Amériques. Ottawa:

Éditions de la Chaire de recherche de l'Université d'Ottawa: Canada: enjeux sociaux et culturels dans une société du savoir, 2012.

JULIEN, Anne-Yvonne, Dir., avec la coll. d'André MAGORD. Littératures québécoise et acadienne. Au prisme de la ville. Rennes: Presses Universitaires de Rennes, 2014.

KOKIS, Serge. Le pavillon des miroirs. Montréal: Lévesque éditeur, 1994.

LABELLE, Maude. «Les lieux de l'écriture migrante. Territoire, mémoire et langue dans Les Lettres chinoises de Ying Chen», Globe, Revue internationale d'études québécoises, vol. 10, no. 1, 2007, p. 43-58.

LAFERRIÈRE, Dany. «Est-il possible d'aller n'importe où, Lise?», dans Lise Gauvin, 
Dir. Les littératures de langue française à l'heure de la mondialisation, Montréal: Hurtubise HMH, 2010, p. 95-96.

LAFERRIÈRE, Dany. Comment faire l'amour avec un nègre sans se fatiguer. Montréal: VLB éditeur, 1985.

LAFERRIÈRE, Dany. Tout ce qu'on ne te dira pas, Mongo. Montréal: Mémoire d'encrier, 2015.

LAFERRIÈRE, Dany. L'énigme du retour. Paris: Grasset, 2009.

LAROSE, Jean. «Transculturation: naissance d'un mot», ViceVersa, no. 21, novembre 1987, p. 9-20.

MAILLET, Antonine. Pélagie-la-Charrette. Paris: Grasset, 1922.

NEPVEU, Pierre et Gilles MARCOTTE, Dir. Montréal imaginaire. Ville et littérature. Montréal, Fides, 1992.

NORMANDIN, Pierre André. «Une nouvelle publicité de Montréal retirée pour son manque de diversit ». La Presse. 29 avril 2017. URL: http://www.lapresse.ca/actualites/ grand-montreal/201704/28/01-5092788-une-nouvelle-publicite-de-montreal-retireepour-son-manque-de-diversite.php (page consultée le 30 octobre 2017).

OUELLET, Pierre. L'esprit migrateur. Montréal: VLB éditeur, 2005.

PROULX, Monique. Ce qu'il reste de moi. Montréal: Boréal, 2015.

ROBIN, Régine Le Cheval blanc de Lénine. Montréal: XYZ éditeur, 1979.

ROBIN, Régine. Nous autres, les autres. Montréal: Boréal, 2011.

ROBIN, Régine. La Québécoite. Montréal: Québec Amérique, 1983.

ROQUEBRUNE, Robert de. Quartier Saint-Louis. Montréal: Fides, 1966.

ROY, Gabrielle. La Route d'Altamont. Montréal: Boréal, 1966.

ROY, Gabrielle. Bonheur d'occasion. Montréal: Boréal, 2009.

SEGURA, Mauricio. Côte-des-Nègres. Montréal: Boréal, 1998.

TASSINARI, Lamberto. «Sens de la transculture», dans Anna Paola Mossetto, Dir., avec la collaboration de Jean-François Plamondon. Le Projet transculturel de Vice Versa. Actes du séminaire international du CISQ à Rome, 25 novembre 2005. Bologne: Pendagron, 2006, p. 61-83.

THÚY, Kim. Ru. Montréal: Libre Expression, 2009.

\section{Notes}

1 Professeure agrégée à l'Université de Winnipeg, Canada, où enseigne les littératures française et francophone des 20e et 21e siècles. a.balint@uwinnipeg.ca

${ }^{2}$ Au sujet de la représentation de la ville en littérature, voir: Anne-Yvonne Julien (dir.), avec la coll. d'André 
Magord, Littératures québécoise et acadienne. Au prisme de la ville. Rennes, Presses Universitaires de Rennes, 2014.

${ }^{3}$ URL: http://www.banq.qc.ca/collections/collections_patrimoniales/bibliographies/lire_montreal/culture1. html (page consultée le 30 octobre 2017).

${ }^{4}$ Régine Robin, Nous autres, les autres, Montréal, Boréal, 2011, p. 304.

${ }_{5}^{5}$ Édouard Glissant, Poétique de la relation, Paris, Gallimard, 1990.

${ }^{6}$ Régine Robin, Nous autres, les autres, op. cit., p. 279.

${ }^{7}$ Lamberto Tassinari, «Sens de la tranculture», dans Anna Paola Mossetto (dir.), avec la collaboration de Jean-François Plamondon, Le Projet transculturel de Vice Versa, Actes du séminaire international du CISQ à Rome, 25 novembre 2005, Bologne, Pendagron, 2006, p. 81.

${ }^{8}$ Patrick Imbert (dir.), Le transculturel et les littératures des Amériques, Ottawa: Éditions de la Chaire de recherche de l'Université d'Ottawa: Canada: enjeux sociaux et culturels dans une société du savoir, 2012, p. 11.

${ }^{9}$ Voir Gilles Deleuze: Différence et répétition, Paris, Presses universitaires de France, 1968.

${ }^{10}$ Jean Larose, «Transculturation: naissance d'un mot », ViceVersa, no. 21, novembre 1987, p. 19.

${ }^{11}$ Pierre Ouellet, L'esprit migrateur, Montréal, VLB éditeur, 2005.

12 Ibid., p. 12.

${ }^{13}$ Maude Labelle, «Les lieux de l'écriture migrante. Territoire, mémoire et langue dans Les Lettres chinoises de Ying Chen», Globe, Revue internationale d'études québécoises, vol. 10, no. 1, 2007, p. 51.

14 Dany Laferrière, «Est-il possible d'aller n'importe où, Lise?», dans Lise Gauvin (dir.), Les littératures de langue française à l’heure de la mondialisation, Montréal, Hurtubise HMH, 2010, p. 95-96.

${ }_{15}$ Simon Harel, Les Passages obligés de l'écriture migrante, Montréal, XYZ éditeur, 2005, p. 228.

${ }^{16}$ Le terme est souvent dévalorisé. Lamberto Tassinari dénigre la notion de la façon suivante: «Je crois que la diversité est un concept galvaudé et mal utilisé par les libertaires eux-mêmes. Au lieu de s'inspirer d'une philosophie de l'autonomie, leur conception de l'individu demeure trop proche de celle qui a été à l'origine de l'individualisme marchand...», dans Fulvio Caccia (dir.), La Transculture et Vice Versa, Montréal, Triptyque, 2010, p. 134.

${ }^{17}$ URL: http://www.lapresse.ca/actualites/montreal/201704/28/01-5092788-une-nouvelle-publicite-demontreal-retiree-pour-son-manque-de-diversite.php (page consultée le 30 octobre 2017). 